\title{
Chirped ordered pulses for ultra-broadband ESR spectroscopy
}

Cite as: J. Chem. Phys. 154, 094201 (2021); https://doi.org/10.1063/5.0038511

Submitted: 23 November 2020 . Accepted: 12 February 2021 . Published Online: 03 March 2021

Jean-Baptiste Verstraete, (D) William K. Myers, and (D) Mohammadali Foroozandeh

COLLECTIONS

Paper published as part of the special topic on 2021 JCP Emerging Investigators Special Collection

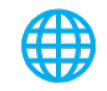

\section{Challenge us.}

What are your needs for periodic signal detection?

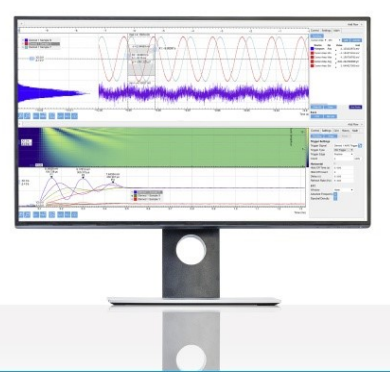

Zurich

Instruments 


\title{
Chirped ordered pulses for ultra-broadband ESR spectroscopy
}

\author{
Cite as: J. Chem. Phys. 154, 094201 (2021); doi: 10.1063/5.0038511 \\ Submitted: 23 November 2020 - Accepted: 12 February 2021 • \\ Published Online: 3 March 2021
}

\author{
Jean-Baptiste Verstraete, ${ }^{1}$ William K. Myers, ${ }^{2, a)}$ (D) and Mohammadali Foroozandeh
}

\begin{abstract}
AFFILIATIONS
${ }^{1}$ Chemistry Research Laboratory, University of Oxford, Mansfield Road, Oxford OX1 3TA, United Kingdom

${ }^{2}$ Centre for Advanced ESR, Inorganic Chemistry Laboratory, University of Oxford, South Parks Road, Oxford OX1 3QR, United Kingdom
\end{abstract}

Note: This paper is part of the 2021 JCP Emerging Investigators Special Collection.

a) Authors to whom correspondence should be addressed: william.myers@chem.ox.ac.uk

and mohammadali.foroozandeh@chem.ox.ac.uk. URL: http://foroozandeh.chem.ox.ac.uk/

\begin{abstract}
Recently, applications of swept-frequency pulses proved to be a useful approach to circumvent the problem of limited excitation bandwidth in pulsed ESR posed by conventional pulses. Here, we present a chirped excitation sequence, CHirped ORdered pulses for Ultra-broadband Spectroscopy (CHORUS), for ultra-broadband ESR spectroscopy. It will be demonstrated that the application of this sequence can address the problems of excitation non-uniformity and sensitivity to instrumental instabilities to a greater extent compared to the current state of the art. This sequence is highly promising for finding applications beyond single excitation in many ESR experiments. Theoretical and experimental results for the proposed method are presented along with calibration strategies for experimental implementation.
\end{abstract}

Published under license by AIP Publishing. https://doi.org/10.1063/5.0038511

\section{INTRODUCTION}

In magnetic resonance, the design of pulses with a wide excitation bandwidth and reduced sensitivity to instrumental imperfections is of great interest. On nuclear magnetic resonance (NMR) and electron spin resonance (ESR) spectrometers, the limited available radio frequency and microwave power poses a problem to the excitation of signals over a very wide range of resonance offsets. In multi-pulse experiments, this cumulative imperfection deteriorates the performance even further. ESR spectroscopy will greatly benefit from the design and application of ultra-broadband pulses as the problem of limited excitation bandwidth is much more serious in pulsed ESR spectroscopy and affects the majority of experiments. ${ }^{1}$ These developments are even more timely, given the increasing demand for broadband multi-pulse experiments, progress in the design of electronic hardware, the advent of arbitrary waveform generators (AWG) for modern ESR instruments, and recent advances in using swept-frequency pulses for broadband excitation. ${ }^{2,3}$
Main approaches to design broadband experiments in magnetic resonance comprise the use of composite pulses, ${ }^{4-10}$ optimal control theory, ${ }^{11-18}$ and swept frequency pulses. ${ }^{19-24}$ In general, a wider excitation bandwidth can be achieved with swept-frequency pulses compared to the two former methods. ${ }^{22}$ In addition, methods combining the use of swept-frequency pulses and optimal control techniques have been reported in the literature.

Applications of swept-frequency pulses for inversion and refocusing have been covered extensively in the literature and are now being implemented in ESR. ${ }^{2,26-28}$ Pulses for broadband inversion have been reported, such as in dipolar spectroscopy with the double electron-electron resonance (DEER) pump pulse. ${ }^{29-32}$ The use of swept-frequency pulses for broadband excitation has also been explored, but to a lesser extent. ${ }^{1}$

Here, we present an implementation of the CHORUS (CHirped ORdered pulses for Ultra-broadband Spectroscopy) sequence $e^{22,24}$ for ultra-broadband excitation in ESR spectroscopy. This comprises a straightforward calibration procedure, measurement of the experimental excitation profile, and assessment 
of the performance via comparison with the current state of the art.

\section{THEORY}

\section{A. Swept-frequency pulses}

It is typical to write the general form of swept-frequency pulses as

$$
S(t)=\omega_{1}(t) \exp (\mathrm{i} \phi(t)),
$$

where the amplitude envelope $\omega_{1}(t)$ and the phase $\phi(t)$ are continuous real-valued functions of time. The relationship between the frequency sweep function $\omega(t)$ and phase $\phi(t)$ of a pulse with duration $\tau_{\mathrm{p}}$ can be written as $\phi(t)=\int_{0}^{\tau_{\mathrm{p}}} \omega(t) d t$.

In the work presented here, chirped pulses with linear frequency sweep were used, i.e., $\omega(t)$ varies linearly and, consequently, $\phi(t)$ varies quadratically with time. ${ }^{42,43}$ The amplitude of such pulses $\left(v_{1}=\gamma B_{1} / 2 \pi\right)$ can be calculated using three parameters as

$$
v_{1}=\sqrt{\frac{\Delta F \mathcal{Q}}{2 \pi \tau_{\mathrm{p}}}},
$$

where $\Delta F$ is the pulse bandwidth and $\mathcal{Q}$ is the adiabaticity factor, a measure for the efficiency of the population inversion in a twolevel system, defined as $\mathcal{Q}(t)=\frac{\omega_{\text {eff }}(t)}{|\mathrm{d} \theta / \mathrm{d} t|}$. Here, $\omega_{\text {eff }}(t)$ is the effective field and $\theta$ is the angle between the effective field and the applied field $\omega_{1} \cdot{ }^{44}$ Approaching the resonance offset, $\omega_{\text {eff }}(t)$ and $|\mathrm{d} \theta / \mathrm{d} t|$ reach their minimum and maximum, respectively, and $\mathcal{Q}$ used in Eq. (2) is achieved. A relationship between the $\mathcal{Q}$ factor and pulse flip angle $(\alpha){ }^{26}$ using an approximated solution of the Landau-Zener formula, ${ }^{45-48}$ can be written as

$$
\mathcal{Q}=\frac{2}{\pi} \ln \left(\frac{2}{\cos (\alpha)+1}\right) .
$$

The effective flip angle of a chirped pulse approaches $180^{\circ}$ asymptotically as the $B_{1}$ increases, and therefore, a value of $\mathcal{Q}$ factor (5 for most practical purposes ${ }^{49}$ or sometimes lower ${ }^{50}$ ) is chosen as a threshold in order to satisfy the adiabatic condition with an affordable $B_{1}$ amplitude. Equation (3) shows that for a $90^{\circ}$ chirped pulse, $\mathcal{Q}$ is fixed and equal to 0.44 . $^{2}$

Two other factors deserve close attention when designing a chirped pulse: (i) time resolution, $\delta \tau$, that determines the sampling rate of the chirped waveform and therefore should be small enough to satisfy the Nyquist condition with respect to the highest frequency of the waveform $(\Delta F / 2)$, although, in practice, the hardware dictates the minimum $\delta \tau$ available and (ii) time-bandwidth product (TBP $=\tau_{\mathrm{p}} \Delta F$, also known as duration-bandwidth product or $\mathrm{R}$-factor) that is an indication of the "chirpiness," or "modulation depth," of a chirped pulse and is used in various domains such as radars, acousto-optics, ${ }^{52}$ optics, ${ }^{53}$ MRI, ${ }^{54-58}$ and NMR. ${ }^{59,60}$ A low value of TBP shows that the pulse does not have enough phase modulation to achieve its desired bandwidth. This corresponds to inversion/excitation profiles presenting hindered uniformity through distortions and ripples ${ }^{61}$ (see Fig. S1 of the supplementary material).

\section{B. Spin-dynamics simulations}

For a single spin- $\frac{1}{2}$, the basis set can be written using Pauli matrices $\left(\sigma_{\alpha}, \alpha \in\{x, y, z\}\right)$ with the Cartesian basis set $\mathcal{L}=\left\{\sigma_{x}, \sigma_{y}, \sigma_{z}\right\}$.
The Hamiltonian of a system consisting of a single spin- $\frac{1}{2}$ with resonance offset $\Omega$ under a chirped pulse can be written as

$$
\mathcal{H}(t)=C_{x}(t) \sigma_{x}+C_{y}(t) \sigma_{y}+\Omega \sigma_{z},
$$

where $C_{x}(t)=\omega_{1}(t) \cos (\phi(t)), C_{y}(t)=\omega_{1}(t) \sin (\phi(t))$, and $\phi(t)$ $=\frac{\pi \Delta F\left(t-\frac{\tau_{\mathrm{p}}}{2}\right)^{2}}{\tau_{\mathrm{p}}}$.

Evolution of the density matrix, $\rho(t)$, can be computed via the solution of the Liouville-von Neumann equation,

$$
\frac{\partial \rho(t)}{\partial t}=-\mathrm{i}[\mathcal{H}(t), \rho(t)] .
$$

The density matrix can be expressed as

$$
\rho(t)=v_{1}(t) \sigma_{x}+v_{2}(t) \sigma_{y}+v_{3}(t) \sigma_{z},
$$

where $\mathbf{v}(t)=\left[v_{1}(t), v_{2}(t), v_{3}(t)\right]^{\top} \in \mathbb{R}^{3}$ is a time-dependent unit vector representing the position of the spin on a Bloch sphere. Using Eqs. (4) and (6), Eq. (5) can be transformed from $\mathrm{SU}(2)$ to $\mathrm{SO}(3)$,

$$
\left(\begin{array}{l}
\dot{v}_{1}(t) \\
\dot{v}_{2}(t) \\
\dot{v}_{3}(t)
\end{array}\right)=\left(\begin{array}{ccc}
0 & -\Omega & C_{y}(t) \\
\Omega & 0 & -C_{x}(t) \\
-C_{y}(t) & C_{x}(t) & 0
\end{array}\right)\left(\begin{array}{l}
v_{1}(t) \\
v_{2}(t) \\
v_{3}(t)
\end{array}\right) .
$$

Considering piece-wise constant propagation, the solution of (7) can be expressed as $\mathbf{v}(t+\delta \tau)=\mathcal{R}_{n} \mathbf{v}(t)$, where

$$
\mathcal{R}_{n}=\exp \left[\left(\begin{array}{ccc}
0 & -\Omega & C_{y, n} \\
\Omega & 0 & -C_{x, n} \\
-C_{y, n} & C_{x, n} & 0
\end{array}\right) \delta \tau\right] .
$$

Here, $C_{x, n}$ and $C_{y, n}$ refer to amplitudes of the $n$th segment of the discretized Cartesian components of the pulse, $C_{x}(t)$ and $C_{y}(t)$. The exponential of a skew-symmetric matrix $\Lambda$ can be expressed using the Rodrigues formula,

$$
e^{\Lambda}=1+\frac{\sin \lambda}{\lambda} \Lambda+\frac{1-\cos \lambda}{\lambda^{2}} \Lambda^{2},
$$

where $\lambda=\delta \tau \sqrt{C_{x, n}^{2}+C_{y, n}^{2}+\Omega^{2}}$.

Therefore, the state of the spin after a pulse consisting of $N$ segments will be $\mathbf{v}\left(\tau_{\mathrm{p}}\right)=\mathcal{R}_{N} \mathcal{R}_{N-1} \ldots \mathcal{R}_{2} \mathcal{R}_{1} \mathbf{v}(0)$. It turns out that this approach is $\sim 3$ times faster than the previous implementation relying on orthogonal rotation matrices.

\section{Insensitivity to $B_{1}$ variations}

Variation of the $B_{1}$ field can deteriorate the performance of the experiment in terms of both the excitation amplitude and phase consistency. Signal loss due to phase variations caused by the changes in $B_{1}$ across the cavity can be mitigated by designing pulses or composite events minimizing the overall $B_{1}$-induced phase variations,

$$
\varphi_{t=T} \propto \int_{0}^{T} \mathbf{B}_{1, \mathrm{eff}} d t,
$$

where $\mathbf{B}_{1, \text { eff }}=\mathbf{B}_{1}^{0}+\delta \mathbf{B}_{1}(\mathbf{r}, t, v)$. Here, $\delta \mathbf{B}_{1}(\mathbf{r}, t, v)$ represents variations in the nominal field with spatial, temporal, and frequential dependencies. Discarding stochastic variation of $B_{1}$, coherent systematic changes will be governed by the geometry and frequency response of the resonator. 


\section{EXPERIMENTAL}

\section{A. Samples}

For excitation profile characterization at the X-band, $\sim 9.45$ $\mathrm{GHz}$, a single-point $\mathrm{x}$-LiPc (Scheme 1.1) sample is prepared under vacuum with a total volume of $0.25 \mathrm{~cm}^{3}$. $\mathrm{x}-\mathrm{LiPc}$ is a microcrystalline powder of the $\mathrm{P} 4 \mathrm{~mm}$ space group in which electrons are delocalized over columnar lithium phthalocyanine stacks with a g-value of $2.00226 \pm 0.00002 .{ }^{64}$ At $294 \mathrm{~K}$, the Fourier transform linewidth is $200 \mathrm{kHz}$, compared to the CW-ESR peak-to-peak linewidth of $4.5 \mu \mathrm{T}$. In relaxation, the $T_{1 e}$ was $4.5 \mu$ s and the phase memory time, $T_{\mathrm{m}}$, was $2.3 \mu \mathrm{s}$, with a stretched exponential value $x=0.88$.

For application examples, 2,6-bis[(((2,2,5,5-tetramethyl-1oxypyrrolin3-yl)-carboxyl)oxy)]-anthracene ${ }^{65}$ (Scheme 1.2), a bisnitroxide, was used for measurements at the Q-band, $\sim 33.8 \mathrm{GHz}$. The sample was dilute in a polymer matrix and filled the resonator height at $1 \mathrm{~mm}$ I.D. For measurements of the sample at $80 \mathrm{~K}, T_{1 e}$ was $188 \mu \mathrm{s}$ and the phase memory time, $T_{\mathrm{m}}$, was $1.46 \mu \mathrm{s}$ with a stretch value $x=0.78$.

\section{B. Hardware}

All measurements were performed on a Biospin EleXSys II E580 Bruker spectrometer. An Applied Systems Engineering (ASE) model 117 TWT amplifier was used with the resonator Bruker BioSpin ER4118-MS2-W1 at the X-band. At Q-band, a dielectric TE01 $\delta$ resonator Bruker EN 5107D2 was used. The Q-band TWTA was an ASE model 187Ka (L3 Electron Devices, Torrance, CA, model 8922HP-1 TWT). Cryogenic liquid helium flow was held at $50 \mathrm{~K}$ with an Oxford Instruments (Abingdon, United Kingdom) CF935O cryostat and Mercury instrument temperature controller.

Pulses were generated by a SpinJet AWG based on an SPDevices (Linköping, Sweden) SDR14 PCI board with a $0.625 \mathrm{~ns}$ time resolution and a sampling rate of 1.6 gigasamples per second (GS/s). Although, theoretically, this sampling rate should allow a uniform emission bandwidth of $1.6 \mathrm{GHz}$, the practical analog limits are its

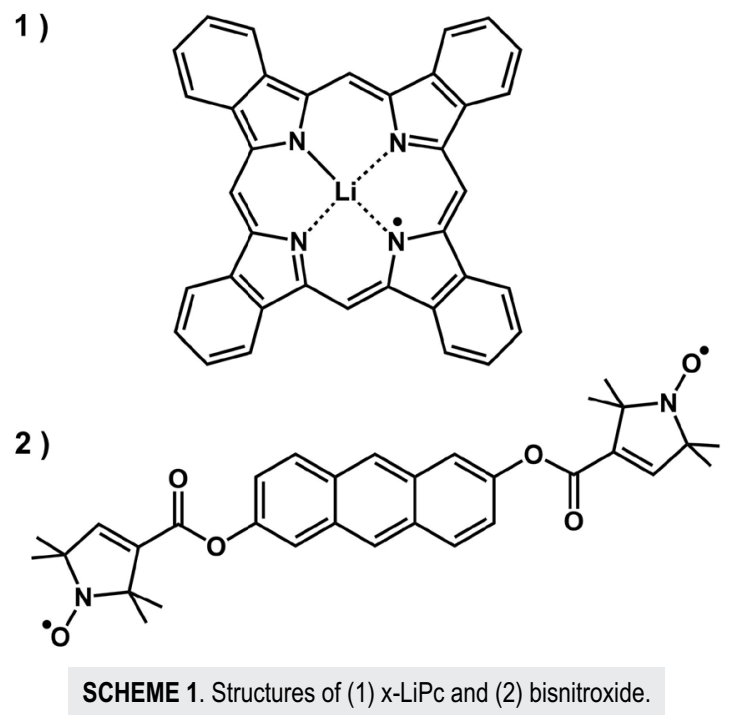

$-1 \mathrm{~dB}$ bandwidth (10\% amplitude variation) going up to $180 \mathrm{MHz}$ and its $-3 \mathrm{~dB}$ bandwidth (30\% amplitude variation) going up to $330 \mathrm{MHz}$.

The upgraded Bruker BioSpin SpecJet III and video amplifier (VAMP III) receiver comprise a $1 \mathrm{GHz}$ bandwidth. A combined AWG/receiver profile is shown in Fig. S4a of the supplementary material.

The resonator profiles were acquired at the X-band and Q-band with a nutation experiment ${ }^{66}$ (see Figs. S4b and S4c of the supplementary material) and served for the generation of the resonatorcompensated pulses.

\section{Pulse generation}

The process of optimal compression of the pulse sequences and selection of parameters is discussed in Sec. IV B. One restriction posed by the spectrometer hardware was that the duration of all pulses must be rounded to the nearest even integer in ns. Parameters used for the generation of the sequences are presented in Table I.

All shaped pulses were generated with functions written in Matlab R2019b, and the EASYSPIN package (5.2.28) ${ }^{67}$ was used to generate the resonator-compensated pulses. Simulations of the excitation profiles $\left[v_{1}, v_{2}\right.$, and $v_{3}$ in Eq. (7), corresponding to $x, y$, and $z$ components of the magnetization, respectively] were carried out according to Sec. II B using Rodrigues rotation matrices [Eq. (9)].

\section{Experiments}

Methods used for the calibration of sequences are presented in Sec. IV C. A 16-steps phase cycle was applied to the KunzBodenhausen sequence, and a 64-step phase cycle was applied to CHORUS (full details are available in Sec. IV of the supplementary material). Correction for the DC leakage at the transmitter frequency was applied by adjusting the output of the AWG I/Q channels.

TABLE I. Parameters used at the X-band and Q-band for the CHORUS ( $\mathrm{CH}$.) and the Kunz-Bodenhausen (K-B.) sequences. $\mathcal{Q}_{\pi}$ indicates the adiabaticity factor for the $\pi$ pulses. TBP $\min$ is the minimum TBP acceptable for compression of the pulses. The effective bandwidth, $\Delta F_{\text {eff }}$, refers to the target bandwidth, $\Delta F$, with smoothed extremities discarded (complete lists of parameters are available in Tables S1 and S2 of the supplementary material).

\begin{tabular}{|c|c|c|c|c|}
\hline Parameter & \multicolumn{2}{|c|}{ X-band } & \multicolumn{2}{|c|}{ Q-band } \\
\hline$v_{1, \max }(\mathrm{MHz})$ & \multicolumn{2}{|c|}{39.89} & \multicolumn{2}{|c|}{37.32} \\
\hline$\Delta F(\mathrm{MHz})$ & \multicolumn{2}{|c|}{400} & \multicolumn{2}{|c|}{350} \\
\hline$Q_{\pi}$ & \multicolumn{2}{|c|}{4} & \multicolumn{2}{|c|}{3} \\
\hline $\mathrm{TBP}_{\min }$ & \multicolumn{2}{|c|}{32} & \multicolumn{2}{|c|}{32} \\
\hline Smoothing & \multicolumn{2}{|c|}{$12.5 \%$} & \multicolumn{2}{|c|}{$15 \%$} \\
\hline$\Delta F_{\text {eff }}(\mathrm{MHz})$ & \multicolumn{2}{|c|}{300} & \multicolumn{2}{|c|}{245} \\
\hline \multirow[t]{2}{*}{$\delta \tau(\mathrm{ns})$} & \multicolumn{2}{|c|}{0.625} & \multicolumn{2}{|c|}{0.625} \\
\hline & $\mathrm{CH}$. & $\mathrm{K}-\mathrm{B}$. & $\mathrm{CH}$. & K-B. \\
\hline$\tau_{\text {seq }}(\mathrm{ns})^{\mathrm{a}}$ & 480 & 640 & 424 & 480 \\
\hline$\tau_{\text {seq+dt }}(n s)^{b}$ & 660 & 640 & 604 & 480 \\
\hline
\end{tabular}

${ }^{\mathrm{a}}$ Total duration of the sequence.

${ }^{\mathrm{b}}$ Total duration of the sequence plus the acquisition dead-time. 
Experiments on the $\mathrm{x}$-LiPc sample were carried out at the $\mathrm{X}$-band and room temperature. The transmitter frequency placed at $9.45 \mathrm{GHz}$. The excitation profiles were obtained with a $560 \mathrm{MHz}$ sweep (200 G), over 41 transmitter offsets ranging from $-280 \mathrm{MHz}$ to $280 \mathrm{MHz}$ in steps of $14 \mathrm{MHz}$, with 8192 points in the freeinduction decay (FID) and 20 averages per scan. The timing of the sequences was adjusted to record half of the echo (pseudo-FID). A 6 ns hard pulse with a four-step phase cycle was used for comparison. The FID was recorded with 16384 points and 10 averages per scan, and it was recorded with 81 points over $400 \mathrm{G}$ field sweep.

Experiments on bisnitroxide were carried out at the Q-band. The transmitter frequency was placed at $33.80 \mathrm{GHz}$ for a refocused echo acquisition at $293 \mathrm{~K}$. The CHORUS sequence echo was recorded with 256 averages per scan and the Kunz-Bodenhausen with 1024 per scan. Both were recorded with 512 points. The conventional hard pulse dataset was acquired with 1024 points and 200 averages per scan using a Hahn-echo experiment consisting of 2 ns and 4 ns pulses, with a 16-step phase cycle. A field-sweep Hahn-echo with 300 points over $200 \mathrm{G}$ and with 1024 points per echo was used as the reference.

The CHEESY (Chirped Echo EPR SpectroscopY) experiment, an electron-electron double resonance detected NMR (EDNMR) method, ${ }^{39}$ was conducted on the bisnitroxide sample at $50 \mathrm{~K}$ with the transmitter frequency placed at $33.95 \mathrm{GHz}$. A new resonator profile was acquired, based on which a resonator-compensated CHORUS sequence was generated. Data were acquired with 512 points and 64 averages per scan in the observed dimension. In the indirect dimension, a $4 \mu$ s high turning angle (HTA) pulse with a Gaussian shape was used. The HTA pulse frequency was varied over 501 offsets ranging from $-250 \mathrm{MHz}$ to $250 \mathrm{MHz}$ in steps of $1 \mathrm{MHz}$.

\section{E. Data analysis}

Post-processing was done in Matlab R2019b and Xepr. The EASYSPIN package $(5.2 .28)^{67}$ was used to import XEPR data to the Matlab environment. At the X-band, the excitation profile was obtained by Fourier transformation of individual FIDs and summation of frequency-domain spectra. The excitation profiles obtained with the $\mathrm{x}$-LiPc sample were phase-corrected using the MatLAB-NMR library. ${ }^{68}$ At the Q-band, the echos obtained with the bisnitroxide sample were zero-filled to 2048 points, and the spectra were phasecorrected using XEPR. The field-sweep spectrum of the bisnitroxide sample was placed into the frequency-domain, centered at 33.8 $\mathrm{GHz}$, and then flipped around the center to be used as the reference spectrum.

The CHEESY dataset was zero-filled to 1024 points and Fourier transformed in the observed dimension. The indirect dimension was interpolated to the same number of points as in the frequencydomain observed dimension. Subsequently, the 2D dataset was sheared, and projections were obtained by summing the resultant $2 \mathrm{D}$ data onto its $x$ - and $y$-axes.

\section{RESULTS AND DISCUSSION}

\section{A. CHORUS sequence}

A single swept-frequency pulse such as a linear chirp is not suitable for broadband excitation. Such a pulse excites signals of different frequencies at different times, in a linear fashion, and

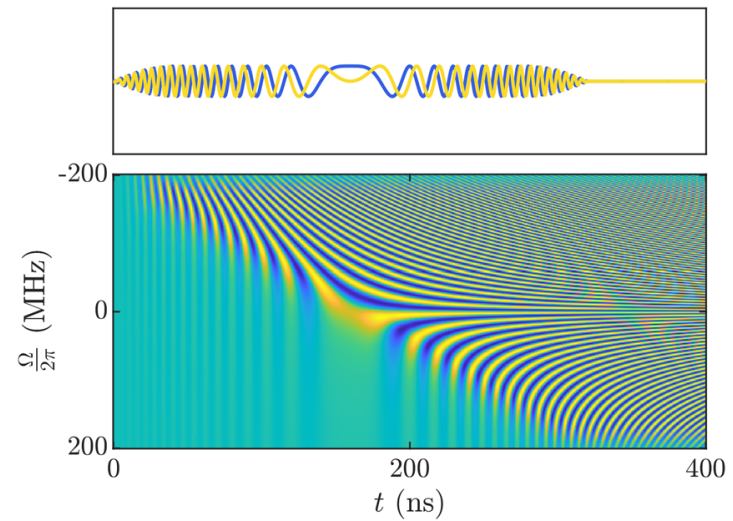

FIG. 1. Illustration of the single chirp excitation pulse: Cartesian components $C_{x}$ and $C_{y}$ (top) and map of the $y$-magnetization as a function of time and offsets simulated on 301 spin- $\frac{1}{2} \mathrm{~s}$ (bottom).

therefore introduces the quadratic phase across the spectrum. This phase problem cannot be corrected with conventional data treatment such as zeroth and first order phase corrections (Fig. 1). However, this observation suggests that an addition of a refocusing pulse with matched duration/bandwidth can solve that offset-dependent phase problem. This technique was introduced by Kunz for imaging $^{19}$ and by Bodenhausen and co-workers for NMR. ${ }^{20,69,70}$ It will be referred to as the Kunz-Bodenhausen sequence in this article.

Although the combination of $90^{\circ}$ and $180^{\circ}$ pulses solves the quadratic phase to a degree, due to a frequency-dependent offresonance irradiation of resonance offsets, it introduces an uncorrectable offset-dependent phase roll, known as the Bloch-Siegert phase shift ${ }^{21,71}$ across the spectrum. This problem can be addressed by the addition of another $180^{\circ}$ pulse to produce a robust selfcompensating sequence, which is less sensitive to $B_{1}$ spatial and frequential inhomogeneities.

The ABSTRUSE (Adjustable, Broadband, Sech/Tanh Rotation Uniform Selective Excitation) sequence introduced by Cano et al. ${ }^{21}$ uses three hyperbolic secant (HS) pulses and was introduced primarily for tunability and selectivity of HS pulses. The CHORUS sequence, ${ }^{22,24}$ on the other hand, uses three linear chirps and offers a much wider excitation bandwidth for a given $B_{1}$ amplitude than ABSTRUSE. CHORUS has been used for ultra-broadband excitation of nuclei with a wide range of chemical shifts (e.g., ${ }^{19} \mathrm{~F}$ and $\left.{ }^{195} \mathrm{Pt}\right) .{ }^{22-24}$ Therefore, the CHORUS sequence is a good choice to effectively address problems with robustness of swept-frequency pulses $^{26}$ with respect to both excitation bandwidth and sensitivity to $B_{1}$ field inhomogeneities in ESR spectroscopy.

It has been demonstrated that by exploiting the additional degree of freedom afforded by the CHORUS scheme, one can construct a compressed sequence that is, counter-intuitively, shorter than the corresponding Kunz-Bodenhausen sequence, ${ }^{24}$ as illustrated in Fig. 2. Here, for the same $B_{1}$ peak amplitude, dictated by the last pulse in the sequence, the CHORUS sequence is $33 \%$ shorter than the Kunz-Bodenhausen sequence, as indicated by the dashed lines in Fig. 2. This is particularly important for ESR where rapid relaxation of the electron spins poses a problem to the implementation of frequency-swept pulses. ${ }^{26}$ The duration of the CHORUS 
a)
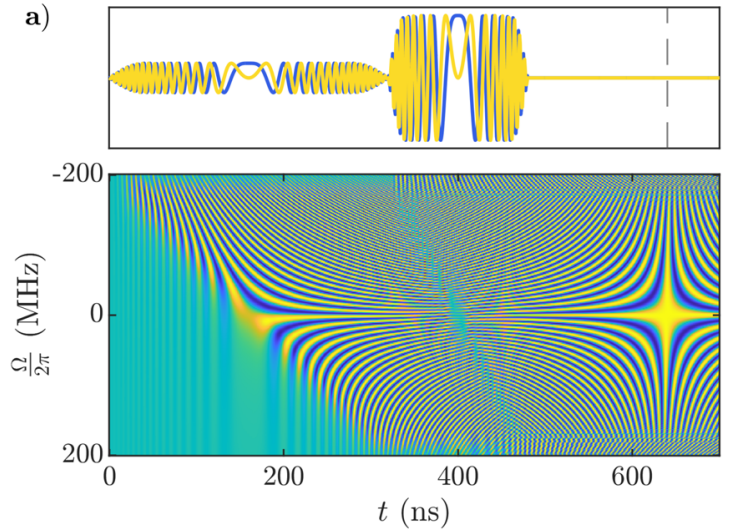

b)
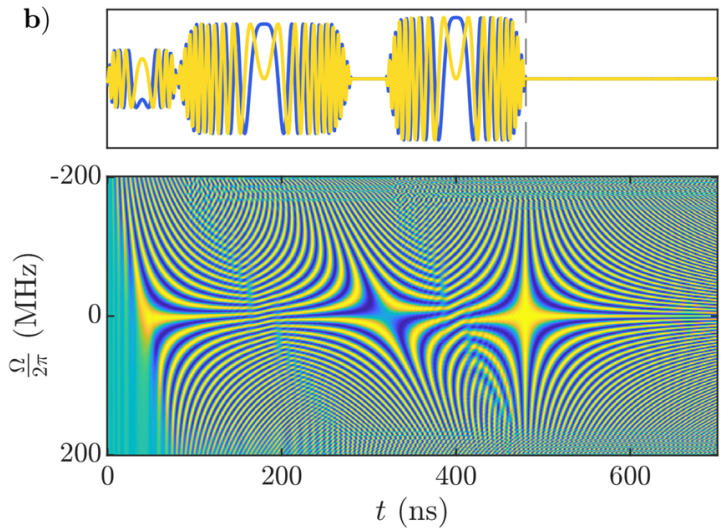

FIG. 2. Illustration of chirped excitation with (a) the Kunz-Bodenhausen and (b) the CHORUS sequences built with the same characteristics and constraints $(X-$ band value in Table I). Both (a) and (b) consist of Cartesian components $C_{x}$ and $C_{y}$ (top) and the map of $y$-magnetization simulated on 301 spin- $\frac{1}{2} s$ with equidistant resonance offsets over $400 \mathrm{MHz}$ bandwidth (bottom). In (a) and (b), the gray dashed line indicates the time at which the refocusing of the signals occurs, and the acquisition is started.

sequence, even increased by the instrument dead time, will be no longer than that of the corresponding Kunz-Bodenhausen sequence, although with superior performance regarding the uniformity of the excitation profile and robustness to instrumental imperfections.

Since the CHORUS sequence utilizes swept-frequency pulses, activation of forbidden transitions is expected, although this may or may not be desirable depending on the nature of a specific application. ${ }^{26}$ In the context of the applications presented in this article, the excitation spectra acquired with the CHORUS sequence did not show any signal loss compared to the ones acquired with the Kunz-Bodenhausen sequence.

\section{B. Sequences design}

One important limitation in pulsed ESR using swept-frequency pulses is the non-linear correspondence between theory and experiment, mainly caused by the microwave components. This effect, which can induce distortion in the effective pulse, can be quantified by recording the resonator profile. ${ }^{18}$ For designing the $\mathrm{CHO}$ RUS sequence, in the first instance, a resonator profile is acquired (see Figs. S4b and S4c of the supplementary material), allowing us to identify a transmitter frequency, a maximum $v_{1}$, and a target bandwidth $\Delta F$. It should be noted that taking the smoothing applied to the amplitude envelope into account, the effective bandwidth of the sequence $\Delta F_{\text {eff }}$ is smaller than the target bandwidth $\Delta F$ (Table I). The adiabaticity factor of the $180^{\circ}$ pulses $Q_{\pi}$ and a minimum $\mathrm{TBP}, \mathrm{TBP}_{\min }$, are then selected accordingly. Including these parameters in Eq. (2) provides the minimum duration (indicated by $\left.{ }^{*}\right)$ for $90^{\circ}$ and $180^{\circ}$ chirped pulses, $\tau_{\frac{\pi}{2}}^{*}$ and $\tau_{\pi}^{*}$, respectively. Finally, the pulse sequence is defined and generated in terms of $\tau_{\frac{\pi}{2}}^{*}$ and $\tau_{\pi}^{*}$. This procedure ensures achieving a maximum compression of the sequence while respecting the constraints imposed by the maximum affordable $B_{1}$ and TBP.

For the Kunz-Bodenhausen sequence, $\tau_{\pi}$ is always the critical pulse duration being shorter than $\tau_{\frac{\pi}{2}}$ with a fixed ratio of $1: 2$, from which the duration of individual elements of the sequence is calculated,

$$
\left[\tau_{\frac{\pi}{2}}, \tau_{\pi}, \tau_{\text {del }}\right]=\left[2 \tau_{\pi}^{*}, \tau_{\pi}^{*}, \tau_{\pi}^{*}\right] .
$$

Therefore, the total duration of the Kunz-Bodenhausen sequence is $4 \tau_{\pi}^{*}$.

For the CHORUS sequence, the additional degree of freedom allows more flexible adjustments of the sequence using both $\tau_{\frac{\pi}{2}}^{*}$ and $\tau_{\pi}^{*}$,

$$
\left[\tau_{\frac{\pi}{2}}, \tau_{\pi(1)}, \tau_{\mathrm{del}}, \tau_{\pi(2)}\right]=\left[\tau_{\frac{\pi}{2}}^{*}, \tau_{\pi}^{*}+\frac{\tau_{\frac{\pi}{2}}^{*}}{2}, \frac{\tau_{\frac{\pi}{2}}^{*}}{2}, \tau_{\pi}^{*}\right] .
$$

Therefore, the total duration of the CHORUS sequence is $2\left(\tau_{\frac{\pi}{2}}^{*}+\tau_{\pi}^{*}\right)$. Note that the CHORUS sequence can be shorter than the Kunz-Bodenhausen sequence by $2\left(\tau_{\pi}^{*}-\tau_{\frac{\pi}{2}}^{*}\right)$, a duration defined by the compression of the $90^{\circ}$ pulse.

To ensure that the adiabaticity factor of $180^{\circ}$ pulses and the TBPs were in compliance with the requirements, spin dynamics simulations were conducted according to the procedure outlined in Sec. II B (see Fig. S2 of the supplementary material). Although the uniformity of the excitation profile was slightly distorted as a result of the applied compression (reduced TBP), the chosen parameters were proven to be experimentally effective.

One experimental limitation, posed by the spectrometer hardware, is the dead time ( $90 \mathrm{~ns}$ on our system) required before triggering the acquisition. For the Kunz-Bodenhausen scheme, this delay can be incorporated in the final delay of the sequence. For the CHORUS sequence, it should be added at the end of the sequence and subsequently between the two $180^{\circ}$ pulses to refocus the echo. Additionally, resonator profiles were used to generate resonatorcompensated pulses to counteract the adverse effect of imperfect microwave components. ${ }^{66,72}$

\section{Calibration}

According to Eq. (10), the adjustment of the relative microwave amplitudes in the sequence can be seen as a simple optimization problem, giving access to the minimum $d \varphi / d B_{1}$ for a range of $v_{1}$ values around the nominal $v_{1}$,

$$
\left\{v_{1, \pi}^{(1)}, v_{1, \pi}^{(2)}\right\}=\underset{v_{1, \pi}^{(1)}, v_{1, \pi}^{(2)}}{\arg \min }\left(\frac{d \varphi}{d v_{1}}\right),
$$


which can be solved offline, as it is done in NMR.

Unfortunately, due to the non-linear response of the hardware on ESR spectrometers (see Fig. S5 of the supplementary material), an input amplitude does not have a linear correlation to the intensity of the corresponding output $B_{1}$ field. In this regard, a series of experimental calibrations were conducted to obtain the effective amplitude percentages that serve as experimental inputs on the spectrometer.

The optimum pulse amplitudes for the Kunz-Bodenhausen sequence were obtained by varying the amplitudes of $90^{\circ}$ and $180^{\circ}$ pulses linearly and independently and choosing a pair of amplitudes corresponding to the maximum magnitude of the signal intensity on the resultant $2 \mathrm{D}$ map. The result of such an experiment is illustrated in Fig. 3(a) for the $\mathrm{x}$-LiPc sample at the X-band. The maximum intensity on the map yielded the experimental amplitude inputs of $14 \%$ and $58 \%$ for $90^{\circ}$ and $180^{\circ}$ pulses, respectively.

The pulse amplitudes for the CHORUS sequence were calibrated in two steps:

(i) The optimum $90^{\circ}$ pulse amplitude was obtained by varying its amplitude in a linear fashion and choosing the one corresponding to the maximum magnitude of the signal intensity (for example, 35\% for the $\mathrm{x}$-LiPc sample at the X-band). For
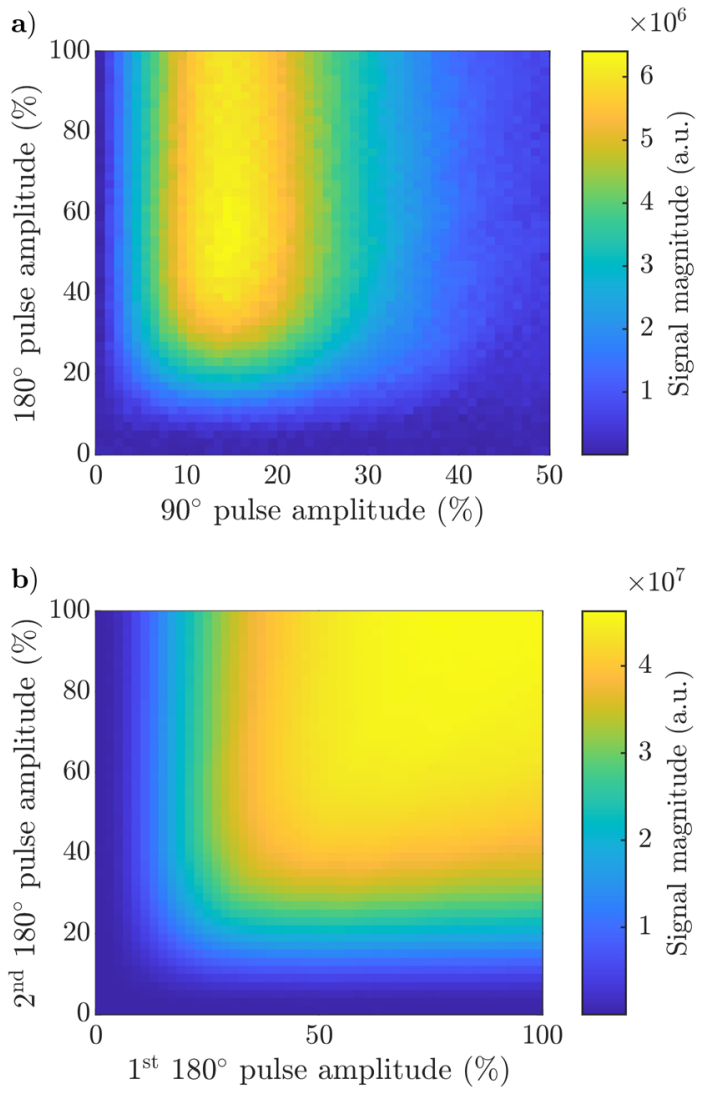

FIG. 3. Experimental 2D map of the signal intensity for calibration of (a) the two pulses of the Kunz-Bodenhausen sequence and (b) the two $180^{\circ}$ pulses of the CHORUS sequence obtained with the X-LiPc sample at the X-band. this experiment, the transmitter was placed $10 \mathrm{G}$ away from the resonance frequency to account for the off-resonance effect.

(ii) The optimum amplitudes of the $180^{\circ}$ pulses were obtained using a similar procedure as of the Kunz-Bodenhausen sequence. Here, the amplitude of the $90^{\circ}$ pulse was kept fixed to the value obtained from step (i) and the amplitudes of two $180^{\circ}$ pulses varied linearly and independently. Consequently, a 2D map of the magnitudes of the signal intensities, presented in Fig. 3(b) for the $\mathrm{x}$-LiPc sample at the X-band, yielded the optimum amplitudes of $80 \%$ and $95 \%$ for $180^{\circ}$ pulses. It should be noted from Fig. 3(b) that once the amplitudes of the $180^{\circ}$ pulses reach a certain threshold, satisfying the required adiabaticity condition, the intensity of the signal increases sharply and reaches a plateau, indicating the successful adiabatic refocusing of the signal.

\section{Experiments with the $\mathrm{X}$-LiPc sample at X-band}

In NMR, experimental inversion/excitation profiles are generally obtained by varying the transmitter offset over a frequency range to emulate the effect of the pulse at different offsets. This is commonly done using a sample with a single narrow line, where the collective response is expected to be a reliable representative of the effective excitation profile of the pulse, or pulse sequence, over a desired bandwidth. ${ }^{21,22,24,69-71,73}$

Although not as widespread as in NMR, a similar approach can be used in ESR. For example, the use of fluoranthenyl hexafluorophosphate $e^{18}$ and $\gamma$-irradiated Herasil glass $\operatorname{rod}^{26}$ has been reported in the literature. Alternatively, the excitation profile can be obtained using an echo integral. ${ }^{33}$ Here, we used Lithium Phthalocyanine (x-LiPc, Scheme 1.1), with a reported ESR half width at half maximum of $150 \mathrm{kHz}-450 \mathrm{kHz}$, in powder form, at X-band, and at room temperature. ${ }^{74}$

The excitation profiles of the conventional hard pulse, the Kunz-Bodenhausen sequence, and the CHORUS sequence, without resonator compensation, on the $\mathrm{x}-\mathrm{LiPc}$ sample at the X-band are presented in Fig. 4. A conventional hard pulse experiment with field sweep acquisition was used as a reference. The CHORUS sequence shows a significantly better uniformity of the excitation profile. The evident intensity variation of the signal (up to $~ 50 \%$ at the extremities, over an effective bandwidth of $300 \mathrm{MHz}$ ) can be linked to various limitations of the hardware, which can distort the excitation profile. The effective pulse, applied to the spins, differs from the digital waveform loaded to the spectrometer software due to the limitations of the Arbitrary Waveform Generator (AWG) ${ }^{72}$ and the microwave components of the spectrometer. ${ }^{18}$ Additionally, the detected signal is bound to the frequency response of the receiver. The combined AWG/receiver profile presents a $17 \%$ variation over the bandwidth of interest (see Fig. S4a of the supplementary material). Application of resonator-compensated pulses did not make any significant difference in the results at the X-band (see Fig. S8 of the supplementary material).

The overall signal intensity for the data collected with the CHORUS sequence is $6 \%$ higher than that with the Kunz-Bodenhausen sequence. This is an indication of the robustness of the CHORUS sequence to the spatial $B_{1}$ inhomogeneities, as shown previously in the NMR literature. ${ }^{22,24}$ 
a)

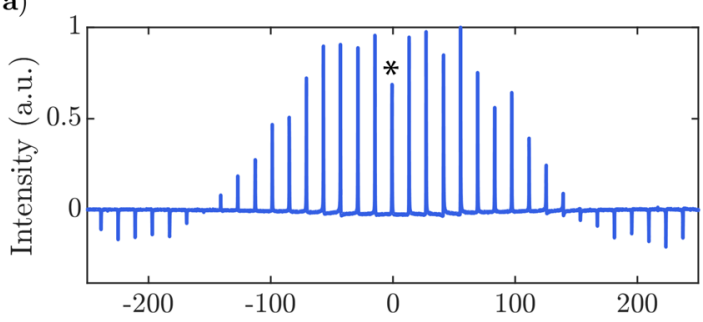

b)

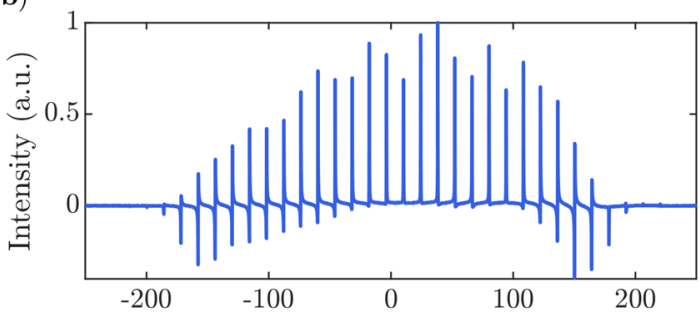

c)

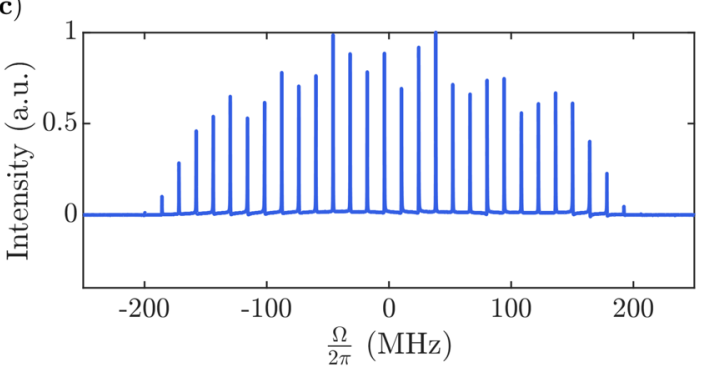

FIG. 4. Experimental excitation profiles at X-band, $9.45 \mathrm{GHz}$, for (a) hard pulse, (b) Kunz-Bodenhausen, and (c) CHORUS sequences. The asterisk indicates a distortion caused by the $\mathrm{DC}$ offset at $0 \mathrm{MHz}$. All spectra are normalized to one.

\section{E. Experiments with the bisnitroxide sample at Q-band}

In order to demonstrate more realistic applications of the proposed scheme, we used the bisnitroxide sample (Scheme 1.2) at the Q-band and at $50 \mathrm{~K}$. Data were acquired using the conventional Hahn echo, the Kunz-Bodenhausen, and the CHORUS sequences, as presented in Fig. 5. A Hahn echo spectrum obtained with field sweep was used as the reference. Resonator compensations were applied to Kunz-Bodenhausen and CHORUS sequences. The performance of the resonator was much more constraining at Q-band compared to X-band, and hence, resonator compensated pulses have significantly improved the quality of the results (see Figs. S4 and S9 of the supplementary material). As shown in Fig. 5(c), the excitation spectrum acquired with the CHORUS sequence is the closest to the reference spectrum. This is due to the robustness of the CHORUS sequence to both frequential and spatial inhomogeneities of the $B_{1}$ field.

The sensitivity of the bisnitroxide data collected with the CHORUS sequence (at global maximum) is $47 \%$ higher than that of the Kunz-Bodenhausen sequence (see Fig. S9 of the supplementary material). The difference is more significant compared to that of the $\mathrm{x}$-LiPc sample at the $\mathrm{X}$-band, as the $B_{1}$ field inhomogeneity is more severe at the Q-band.
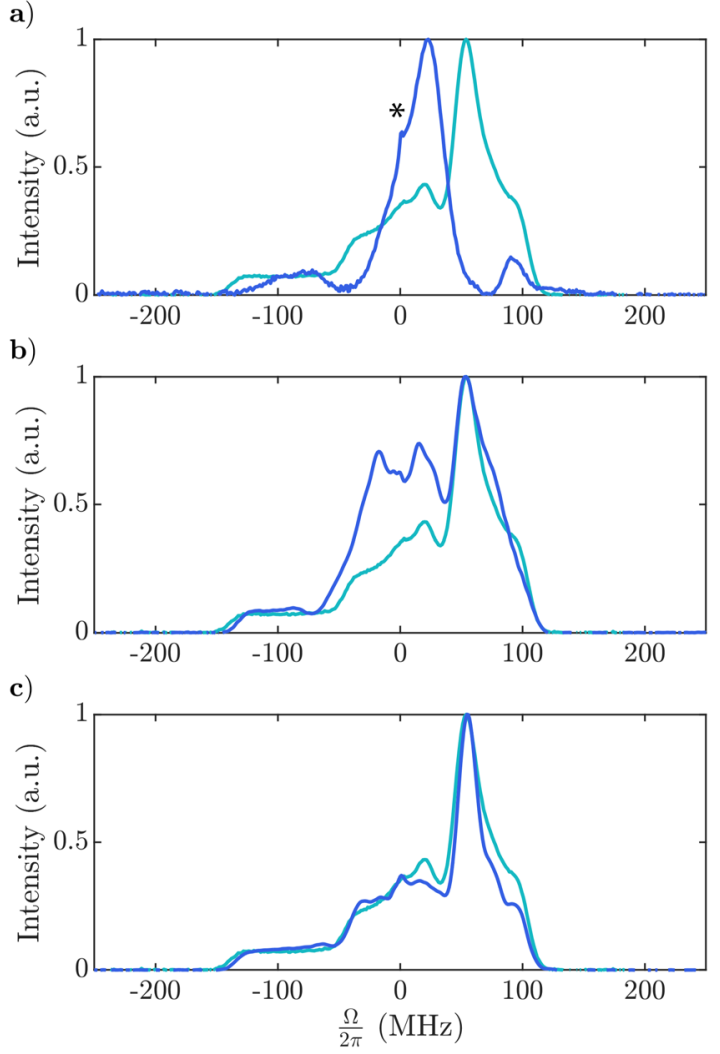

FIG. 5. Spectra of the bisnitroxide sample acquired at Q-band using (a) Hahn echo, (b) Kunz-Bodenhausen, and (c) CHORUS sequences (dark blue). A field sweep reference measurement is indicated in light blue. The asterisk indicates an artifact due to the $\mathrm{DC}$ offset at $\mathrm{OMHz}$. All spectra are normalized to one.

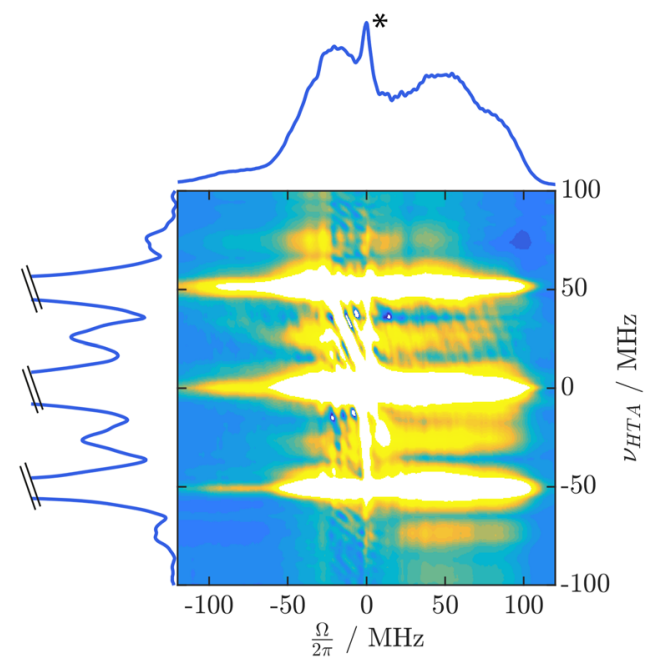

FIG. 6. Magnitude-mode 2D spectrum of the bisnitroxide sample acquired with the CHEESY sequence at $50 \mathrm{~K}$ using the CHORUS sequence in detection. The asterisk indicates an artifact due to the $\mathrm{DC}$ offset at $0 \mathrm{MHz}$. The ${ }^{1} \mathrm{H}$ peaks are truncated to allow for better visualization of the ${ }^{14} \mathrm{~N}$ peaks. 
Figure 6 demonstrates another application for the CHORUS sequence, the CHEESY-detected NMR, which harnesses the broadband nature of chirped echo excitation, to detect data in the observed dimension in one shot. In our application, the CHORUS sequence was used as the read sequence, replacing the Kunz-Bodenhausen sequence presented in the original application. ${ }^{39}$ The CHEESY CHORUS spectrum shows the ${ }^{14} \mathrm{~N}$ nuclear transitions on the $y$-axis projection.

\section{CONCLUSION}

In the present work, we have introduced an ultra-broadband excitation scheme for ESR spectroscopy based on CHORUS (CHirped, ORdered pulses for Ultra-broadband Spectroscopy). It was shown that the proposed method offers a wider and more uniform excitation bandwidth than the current state of the art. Since the limitations of the ESR hardware, including the receiver and resonator bandwidth, hinder the CHORUS sequence from reaching its full potential, future improvements in ESR hardware design should enable an even better performance. Additionally, the CHORUS method could be combined with feedback loop optimization, where multiple aspects of the problems posed by the ESR hardware can be addressed at once. The CHORUS sequence has the potential to find applications in many ESR experiments, especially where experiments already consist of three pulses.

\section{SUPPLEMENTARY MATERIAL}

See the supplementary material for additional experiments, experimental parameters, simulations, and calibration remarks.

\section{ACKNOWLEDGMENTS}

M.F. thanks the Royal Society for a University Research Fellowship and a University Research Fellow Enhancement Award (Grant Nos. URF $\backslash R 1 \backslash 180233$ and RGF $\backslash E A \backslash 181018$ ). W.K.M. and M.F. thank the John Fell OUP Research Fund (Grant No. 0007019). J.-B.V. thanks the EPSRC Doctoral Training Partnership. W.K.M. was supported by the Centre for Advanced ESR, UK EPSRC (Grant Nos. EP/V036408/1 and EP/L011972/1), and the Department of Chemistry.

\section{DATA AVAILABILITY}

All Matlab functions for the generation of chirped pulses and associated simulations and raw datasets and related processing codes for all experiments that support the findings of this study are openly available in GitHub repository, MrCHIRPLAB, via https://github.com/foroozandehgroup/MRChirpLab. ${ }^{76}$

\section{REFERENCES}

${ }^{1}$ A. Schweiger and G. Jeschke, Principles of Pulse Electron Paramagnetic Resonance (Oxford University Press, Oxford, 2001).

${ }^{2}$ P. E. Spindler, P. Schöps, W. Kallies, S. J. Glaser, and T. F. Prisner, "Perspectives of shaped pulses for EPR spectroscopy," J. Magn. Reson. 280, 30-45 (2017).

${ }^{3}$ G. Jeschke, “Quo vadis EPR?," J. Magn. Reson. 306, 36-41 (2019).

${ }^{4} \mathrm{M}$. Levitt and R. Ernst, "Composite pulses constructed by a recursive expansion procedure,” J. Magn. Reson. 55, 247-254 (1983).
${ }^{5}$ A. Shaka and R. Freeman, “Composite pulses with dual compensation," J. Magn. Reson. 55, 487-493 (1983).

${ }^{6}$ M. H. Levitt, D. Suter, and R. R. Ernst, "Composite pulse excitation in three-level systems," J. Chem. Phys. 80, 3064-3068 (1984).

${ }^{7}$ C. Counsell, M. H. Levitt, and R. R. Ernst, "Analytical theory of composite pulses," J. Magn. Reson. 63, 133-141 (1985).

${ }^{8}$ R. Tycko, H. Cho, E. Schneider, and A. Pines, "Composite pulses without phase distortion,” J. Magn. Reson. 61, 90-101 (1985).

${ }^{9}$ M. H. Levitt, “Composite pulses," Prog. Nucl. Magn. Reson. Spectrosc. 18, 61122 (1986).

${ }^{10}$ A. Shaka and A. Pines, "Symmetric phase-alternating composite pulses," J. Magn. Reson. 71, 495-503 (1987).

${ }^{11}$ T. E. Skinner, T. O. Reiss, B. Luy, N. Khaneja, and S. J. Glaser, "Application of optimal control theory to the design of broadband excitation pulses for highresolution NMR," J. Magn. Reson. 163, 8-15 (2003).

${ }^{12}$ K. Kobzar, T. E. Skinner, N. Khaneja, S. J. Glaser, and B. Luy, "Exploring the limits of broadband excitation and inversion pulses," J. Magn. Reson. 170, 236243 (2004).

${ }^{13}$ N. Khaneja, T. Reiss, C. Kehlet, T. Schulte-Herbrüggen, and S. J. Glaser, "Optimal control of coupled spin dynamics: Design of NMR pulse sequences by gradient ascent algorithms," J. Magn. Reson. 172, 296-305 (2005).

${ }^{14}$ B. Luy, K. Kobzar, T. E. Skinner, N. Khaneja, and S. J. Glaser, "Construction of universal rotations from point-to-point transformations," J. Magn. Reson. 176, 179-186 (2005).

${ }^{15}$ T. E. Skinner, T. O. Reiss, B. Luy, N. Khaneja, and S. J. Glaser, "Tailoring the optimal control cost function to a desired output: Application to minimizing phase errors in short broadband excitation pulses," J. Magn. Reson. 172, 17-23 (2005).

${ }^{16}$ K. Kobzar, T. E. Skinner, N. Khaneja, S. J. Glaser, and B. Luy, "Exploring the limits of broadband excitation and inversion: II. Rf-power optimized pulses," J. Magn. Reson. 194, 58-66 (2008).

${ }^{17}$ K. Kobzar, S. Ehni, T. E. Skinner, S. J. Glaser, and B. Luy, "Exploring the limits of broadband $90^{\circ}$ and $180^{\circ}$ universal rotation pulses," J. Magn. Reson. 225, 142-160 (2012).

${ }^{18}$ P. E. Spindler, Y. Zhang, B. Endeward, N. Gershernzon, T. E. Skinner, S. J. Glaser, and T. F. Prisner, "Shaped optimal control pulses for increased excitation bandwidth in EPR," J. Magn. Reson. 218, 49-58 (2012).

${ }^{19} \mathrm{D}$. Kunz, "Use of frequency-modulated radiofrequency pulses in MR imaging experiments," Magn. Reson. Med. 3, 377-384 (1986).

${ }^{20}$ J.-M. Böhlen, M. Rey, and G. Bodenhausen, "Refocusing with chirped pulses for broadband excitation without phase dispersion," J. Magn. Reson. 84, 191-197 (1989).

${ }^{21}$ K. E. Cano, M. A. Smith, and A. J. Shaka, "Adjustable, broadband, selective excitation with uniform phase," J. Magn. Reson. 155, 131-139 (2002).

${ }^{22}$ J. E. Power, M. Foroozandeh, R. W. Adams, M. Nilsson, S. R. Coombes, A. R. Phillips, and G. A. Morris, "Increasing the quantitative bandwidth of NMR measurements," Chem. Commun. 52, 2916-2919 (2016).

${ }^{23}$ J. E. Power, M. Foroozandeh, P. Moutzouri, R. W. Adams, M. Nilsson, S. R. Coombes, A. R. Phillips, and G. A. Morris, "Very broadband diffusionordered NMR spectroscopy: ${ }^{19} \mathrm{~F}$ DOSY," Chem. Commun. 52, 6892-6894 (2016).

${ }^{24}$ M. Foroozandeh, M. Nilsson, and G. A. Morris, "Improved ultra-broadband chirp excitation,” J. Magn. Reson. 302, 28-33 (2019).

${ }^{25}$ A. R. Altenhof, A. W. Lindquist, L. D. D. Foster, S. T. Holmes, and R. W. Schurko, "On the use of frequency-swept pulses and pulses designed with optimal control theory for the acquisition of ultra-wideline NMR spectra," J. Magn. Reson. 309, 106612 (2019).

${ }^{26} \mathrm{G}$. Jeschke, S. Pribitzer, and A. Doll, "Coherence transfer by passage pulses in electron paramagnetic resonance spectroscopy,” J. Phys. Chem. B 119, 1357013582 (2015).

${ }^{27}$ P. E. Spindler, P. Schoeps, A. Bowen, B. Endeward, and T. F. Prisner, "Shaped pulses in EPR," eMagRes 5, 1477-1492 (2016).

${ }^{28}$ T. F. Prisner, "Shaping EPR: Phase and amplitude modulated microwave pulses," J. Magn. Reson. 306, 98-101 (2019). 
${ }^{29}$ P. E. Spindler, S. J. Glaser, T. E. Skinner, and T. F. Prisner, "Broadband inversion PELDOR spectroscopy with partially adiabatic shaped pulses," Angew. Chem., Int. Ed. 52, 3425-3429 (2013).

${ }^{30}$ T. Bahrenberg, Y. Rosenski, R. Carmieli, K. Zibzener, M. Qi, V. Frydman, A. Godt, D. Goldfarb, and A. Feintuch, "Improved sensitivity for W-band Gd(III)Gd(III) and nitroxide-nitroxide DEER measurements with shaped pulses," J. Magn. Reson. 283, 1-13 (2017).

${ }^{31}$ A. Doll, M. Qi, N. Wili, S. Pribitzer, A. Godt, and G. Jeschke, "Gd(III)-Gd(III) distance measurements with chirp pump pulses," J. Magn. Reson. 259, 153-162 (2015).

${ }^{32}$ A. Scherer, S. Tischlik, S. Weickert, V. Wittmann, and M. Drescher, "Optimising broadband pulses for DEER depends on concentration and distance range of interest," Magn. Reson. 1, 59-74 (2020).

${ }^{33}$ A. Doll and G. Jeschke, "Fourier-transform electron spin resonance with bandwidth-compensated chirp pulses," J. Magn. Reson. 246, 18-26 (2014).

${ }^{34}$ T. F. Segawa, A. Doll, S. Pribitzer, and G. Jeschke, "Copper ESEEM and HYSCORE through ultra-wideband chirp EPR spectroscopy," J. Chem. Phys. 143, 044201 (2015)

${ }^{35}$ P. Schöps, P. E. Spindler, A. Marko, and T. F. Prisner, "Broadband spin echoes and broadband SIFTER in EPR,” J. Magn. Reson. 250, 55-62 (2015).

${ }^{36}$ S. Pribitzer, T. F. Segawa, A. Doll, and G. Jeschke, "Transverse interference peaks in chirp FT-EPR correlated three-pulse ESEEM spectra," J. Magn. Reson. 272, 37-45 (2016)

${ }^{37} \mathrm{~A}$. Doll and G. Jeschke, "EPR-correlated dipolar spectroscopy by Q-band chirp SIFTER,” Phys. Chem. Chem. Phys. 18, 23111-23120 (2016).

${ }^{38} \mathrm{~A}$. Doll and G. Jeschke, "Wideband frequency-swept excitation in pulsed EPR spectroscopy,” J. Magn. Reson. 280, 46-62 (2017).

${ }^{39} \mathrm{~N}$. Wili and G. Jeschke, "Chirp echo Fourier transform EPR-detected NMR," J. Magn. Reson. 289, 26-34 (2018).

${ }^{40}$ I. Ritsch, H. Hintz, G. Jeschke, A. Godt, and M. Yulikov, "Improving the accuracy of $\mathrm{Cu}(\mathrm{II})$-nitroxide RIDME in the presence of orientation correlation in water-soluble $\mathrm{Cu}(\mathrm{II})$-nitroxide rulers," Phys. Chem. Chem. Phys. 21, 9810-9830 (2019).

${ }^{41}$ F. D. Breitgoff, K. Keller, M. Qi, D. Klose, M. Yulikov, A. Godt, and G. Jeschke, "UWB DEER and RIDME distance measurements in $\mathrm{Cu}(\mathrm{II})-\mathrm{Cu}(\mathrm{II})$ spin pairs," J. Magn. Reson. 308, 106560 (2019).

${ }^{42}$ S. W. Smith, Digital Signal Processing: A Practical Guide for Engineers and Scientists, Demystifying Technology Series (Newnes, 2003).

${ }^{43}$ P. Flandrin, "Time frequency and chirps," in Wavelet Applications VIII, edited by H. H. Szu, D. L. Donoho, A. W. Lohmann, W. J. Campbell, and J. R. Buss (SPIE-International Society for Optics and Photonics, 2001), Vol. 4391, pp. 161175.

${ }^{44} \mathrm{~J}$. Baum, R. Tycko, and A. Pines, "Broadband and adiabatic inversion of a twolevel system by phase-modulated pulses," Phys. Rev. A 32, 3435-3447 (1985).

${ }^{45}$ L. D. Landau, "Zur theorie der energieubertragung II," Phys. Z. Sowjet 2, 46-51 (1932).

${ }^{46}$ C. Zener and R. H. Fowler, "Non-adiabatic crossing of energy levels," Proc. R. Soc. London, Ser. A 137, 696-702 (1932).

${ }^{47}$ E. Majorana, "Atomi orientati in campo magnetico variabile," Nuovo Cimento 9, 43-50 (1932).

${ }^{48}$ E. C. G. Stueckelberg, "Theory of inelastic collisions between atoms (theory of inelastic collisions between atoms, using two simultaneous differential equations)," Helv. Phys. Acta 5, 369-422 (1932).

${ }^{49} \bar{E}$. Kupce and R. Freeman, "Stretched adiabatic pulses for broadband spin inversion,” J. Magn. Reson., Ser. A 117, 246-256 (1995).

${ }^{50}$ M. Deschamps, G. Kervern, D. Massiot, G. Pintacuda, L. Emsley, and P. J. Grandinetti, "Superadiabaticity in magnetic resonance," J. Chem. Phys. 129, 204110 (2008).

${ }^{51}$ H. Meikle, Modern Radar Systems, 2nd ed., Artech House Radar Library (Artech House, Boston, 2008).
${ }^{52}$ C. S. Tsai, Guided-Wave Acousto-Optics: Interactions, Devices, and Applications (Springer Science \& Business Media, 2013), Vol. 23.

${ }^{53}$ A. E. Siegman, Lasers (University Science Books, Mill Valley, CA, 1986).

${ }^{54} \mathrm{~J}$.-N. Dumez and L. Frydman, "Multidimensional excitation pulses based on spatiotemporal encoding concepts," J. Magn. Reson. 226, 22-34 (2013).

${ }^{55}$ M. A. Bernstein, K. F. King, and X. J. Zhou, Handbook of MRI Pulse Sequences (Academic Press, 2004)

${ }^{56}$ N. Ben-Eliezer, U. Goerke, K. Ugurbil, and L. Frydman, "Functional MRI using super-resolved spatiotemporal encoding," Magn. Reson. Imaging 30, 1401-1408 (2012).

${ }^{57}$ D. Idiyatullin, C. Corum, S. Moeller, and M. Garwood, "Gapped pulses for frequency-swept MRI,” J. Magn. Reson. 193, 267-273 (2008).

${ }^{58}$ V. L. Landes and K. S. Nayak, "Simple method for RF pulse measurement using gradient reversal,” Magn. Reson. Med. 79, 2642-2651 (2018).

${ }^{59} \mathrm{R}$. Bhattacharyya and L. Frydman, "Quadrupolar nuclear magnetic resonance spectroscopy in solids using frequency-swept echoing pulses," J. Chem. Phys. 127, 194503 (2007).

${ }^{60}$ Y. Ke, D. G. Schupp, and M. Garwood, "Adiabatic DANTE sequences for $B_{1}$ insensitive narrowband inversion," J. Magn. Reson. 96, 663-669 (1992).

${ }^{61}$ T. E. Skinner, N. I. Gershenzon, M. Nimbalkar, and S. J. Glaser, "Optimal control design of band-selective excitation pulses that accommodate relaxation and RF inhomogeneity," J. Magn. Reson. 217, 53-60 (2012).

${ }^{62} \mathrm{M}$. Foroozandeh, "Spin dynamics during chirped pulses: Applications to homonuclear decoupling and broadband excitation," J. Magn. Reson. 318, 106768 (2020).

${ }^{63} \mathrm{O}$. Rodrigues, "Des lois géométriques qui régissent les déplacements d'un système solide dans l'espace, et de la variation des coordonnées provenant de ces déplacements considérés indépendamment des causes qui peuvent les produire," J. Math. Pures Appl. 5, 380-440 (1840).

${ }^{64}$ H. Sato, L. A. Dalton, D. Ha, R. W. Quine, S. S. Eaton, and G. R. Eaton, "Electron spin relaxation in $x$-lithium phthalocyanine," J. Phys. Chem. B 111, 7972-7977 (2007).

${ }^{65}$ R. G. Larsen and D. J. Singel, "Double electron-electron resonance spinecho modulation: Spectroscopic measurement of electron spin pair separations in orientationally disordered solids," J. Chem. Phys. 98, 5134-5146 (1993).

${ }^{66}$ A. Doll, S. Pribitzer, R. Tschaggelar, and G. Jeschke, "Adiabatic and fast passage ultra-wideband inversion in pulsed EPR," J. Magn. Reson. 230, 27-39 (2013).

${ }^{67}$ S. Stoll and A. Schweiger, "EasySpin, a comprehensive software package for spectral simulation and analysis in EPR," J. Magn. Reson. 178, 42-55 (2006).

${ }^{68} \mathrm{P}$. Blümler, Matlab NMR-library.

${ }^{69} \mathrm{~J}$. M. Böhlen and G. Bodenhausen, "Experimental aspects of chirp NMR spectroscopy," J. Magn. Reson., Ser. A 102, 293-301 (1993).

${ }^{70}$ V. L. Ermakov, J. M. Bohlen, and G. Bodenhausen, "Improved schemes for refocusing with frequency-modulated chirp pulses," J. Magn. Reson., Ser. A 103, 226-229 (1993).

${ }^{71}$ T.-L. Hwang, P. C. M. van Zijl, and M. Garwood, "Broadband adiabatic refocusing without phase distortion," J. Magn. Reson. 124, 250-254 (1997).

${ }^{72}$ T. Kaufmann, T. J. Keller, J. M. Franck, R. P. Barnes, S. J. Glaser, J. M. Martinis, and S. Han, "DAC-board based X-band EPR spectrometer with arbitrary waveform control," J. Magn. Reson. 235, 95-108 (2013).

${ }^{73}$ V. L. Ermakov and G. Bodenhausen, "Broadband excitation in magnetic resonance by self-refocusing doubly frequency-modulated pulses," Chem. Phys. Lett. 204, 375-380 (1993).

${ }^{74} \mathrm{M}$. Brinkmann, P. Turek, and J.-J. André, "EPR study of the $\mathrm{x}, \alpha$ and $\beta$ structures of lithium phthalocyanine," J. Mater. Chem. 8, 675-685 (1998).

${ }^{75}$ D. L. Goodwin, W. K. Myers, C. R. Timmel, and I. Kuprov, "Feedback control optimisation of ESR experiments," J. Magn. Reson. 297, 9-16 (2018).

${ }^{76}$ J.-B. Verstraete, MRChirpLab, https://github.com/foroozandehgroup/MRChirp Lab, 2020. 